\title{
Leveraging General Collective Intelligence to Increase the Impact of Pattern Analysis and Machine Intelligence Algorithms
}

Andy E. Williams, Nobeah Foundation, Nairobi, Kenya

\begin{abstract}
The emerging science of General Collective Intelligence or GCI describes how GCI as a hypothetical cognitive computing platform might enable groups of individuals or intelligent agents working on behalf of those individuals to self-assemble into a massive network of cooperation. Such networks are predicted to be able to radically increase the speed and scale at which the group might construct platforms from a library of logic based functionality (i.e. procedural program) and/or a library of text pattern recognition based functionality (i.e. machine intelligence algorithms), as well as enable groups to do so on a self-sustaining basis, where these self-assembled platforms are also predicted to achieve significantly better outcomes for the group of users. To facilitate this self-assembly, GCI leverages Human-Centric Functional Modeling to represent such components in terms of paths through a functional state space defined to represent all possible behavior of this cognitive computing platform so that all such components can be represented. This article explores how this same approach might be applied to define cognitive visual processing platforms that radically increase capacity to leverage all existing image processing functionality as well as all visual pattern recognition algorithms in order to radically increase capacity for and effectiveness at image recognition.
\end{abstract}

\section{Keywords:}

General Collective Intelligence, machine intelligence, pattern analysis, functional state space, semantic model

\section{Introduction}

A General Collective Intelligence or GCI [1] is a hypothetical platform that enables individuals, or intelligent agents participating on behalf of each individual, to self-assemble into potentially massive networks of self-sustaining cooperation, where these networks act as a collective cognition capable of executing collective reasoning processes. This massive network applied to that execution is predicted to create the capacity to exponentially increase the speed, scale, and effectiveness of that reasoning. A GCI leverages Human-Centric Functional Modeling or HCFM [2] to define a functional state space that has the capacity to represent all possible behaviors of this collective cognition that occur within the cognitive domain. This collective space of concepts connected by collective reasoning, or "collective conceptual space" that serves as the functional state space for this collective cognition is a graph containing a network of nodes, each node representing a concept, and each concept being a functional state of the collective cognition, where those nodes are connected by edges, with each edge representing a collective reasoning process that collective cognition might take to transition from one concept (one functional state) to another. This collective conceptual space is hypothesized to be a complete semantic representation for concepts and reasoning that might be navigated by the GCI, since all concepts are defined by all possible reasoning connecting them to other concepts, and since all reasoning is defined by all concepts it can possibly be used to connect.

There are a number of properties that conceptual space must have in order for it to provide a complete semantic representation of concepts and reasoning. That any functional state space provides a humancentric representation of the functional states or processes in a given domain of behavior of some system (i.e. provides a fully self-contained representation of human meaning in that functional domain) means that functional state space can be used to define a model capable of storing all the properties of that functional state space at any scale without the need for any external reference or translation table, 
so that any behavior in that domain at any scale can be explored using the same infrastructure used at any other scale. With such a fully self-contained model any process in the functional domain in every simulation, every computer game, every Computer Aided Design (CAD) tool, every Computer Generated Image (CGI), or every other computer interaction, becomes part of a single data format, and every component in every process can potentially be reused by every other such computer interaction. When the functional state space is the conceptual space this means reasoning implemented in one program can potentially be modeled and reused in another. When the functional state space is the visual state space this means object recognition implemented in one program can potentially be modeled and reused in another.

While the collective cognition represented by GCI is predicted to have internal (not consciously observable) behaviors required for its operation, in addition to the external (consciously observable) behaviors described by this collective conceptual space, all observable behaviors of the GCI within the cognitive domain are expected to be confined within this collective conceptual space. Similarly, all consciously observable behaviors that the human visual system possesses are expected to be confined to the visual state space.

\section{Semantic Perception vs Raw Sensory Signals}

Below the conceptual space in the hierarchy of perception is the conceptual field, a field of signals received directly from the neurons. In Human-Centric Functional Modeling each perception that a human might become aware of can be understood in terms of others that are simpler to understand in a given problem domain. This conceptual field is most simply understood by analogy with the sensorymotor field, the of signals received from the sensory receptors (sensory cells) in the body or sent to the motion transmission (muscle or motor cells) in the body. In Human-Centric Functional Modeling this field is represented as coming from an array of sensory receptors and/or being sent to an array of motor cells in the body. These fields have a spatial distribution, a sequence at each point in the spacial distribution, and a pattern in the sequence. Consequently, it is hypothesized that three functions (position, sequence, and pattern) can be used to navigate this entire field.

The sensory-motor field contains raw signals from sensory cells, but on top of the sensory-motor field in the hierarchy of perception is the sensory motor space that contains not just raw and imperceptible electrical signals, but physical sensations that have been perceived from the distributions, sequences, and patterns of signals. By analogy with the sensory-motor field, all other physical senses can also be represented as arising from a field. Hence, the sensory-motor field is hypothesized to be composite field consisting of a tactile field, an olfactory field, an auditory field, a gustatory field, and a visual field, which is related to the subject of this paper. Some of the visual pattern analysis and machine learning algorithms that must be represented in this functional modeling approach occur at the level of converting raw visual signals to a visual state space containing meaningful objects along with their colors, resolution, and other visual properties. Other algorithms that must be represented in this functional modeling approach occur at the level of geometrically shifting the meaning of objects so that any objects that are recognized can be recognized at other positions or orientations in this visual state space. In either case, one of the most powerful aspects of this approach is the opportunity it creates to reuse any insights gained in modeling one human system when it comes to understanding another. In this case, any insights used to categorize algorithms that can help translate the visual field into objects we can "see", are insights that can be used to understand how any sensory-motor, visual, or other sensory perceptions might be received as signals input to our conceptual field, and might be translated into concepts our cognitive system can "see", and therefore can understand.

\section{Extending the Example of Libraries in Conceptual Space to Libraries in Visual Space}


The importance of the individual or collective conceptual space being a complete semantic representation of behavior within the cognitive domain is that we know that individual reasoning consists of either type 1 (intuitive) or type 2 (rational methodical) reasoning [3]. In conceptual space type 1 reasoning is represented by a direct path between one concept or another, where that path is defined by pattern recognition or other processes that might be automated by text based machine learning algorithms. This type of reasoning is believed to be optimal where solutions are uncomputable. Similarly, in conceptual space type 2 reasoning is represented by a stepwise path through intermediate concepts, where each step represents a step of logic that might be automated by a procedural program. This type of reasoning is believed to be optimal where solutions are computable.

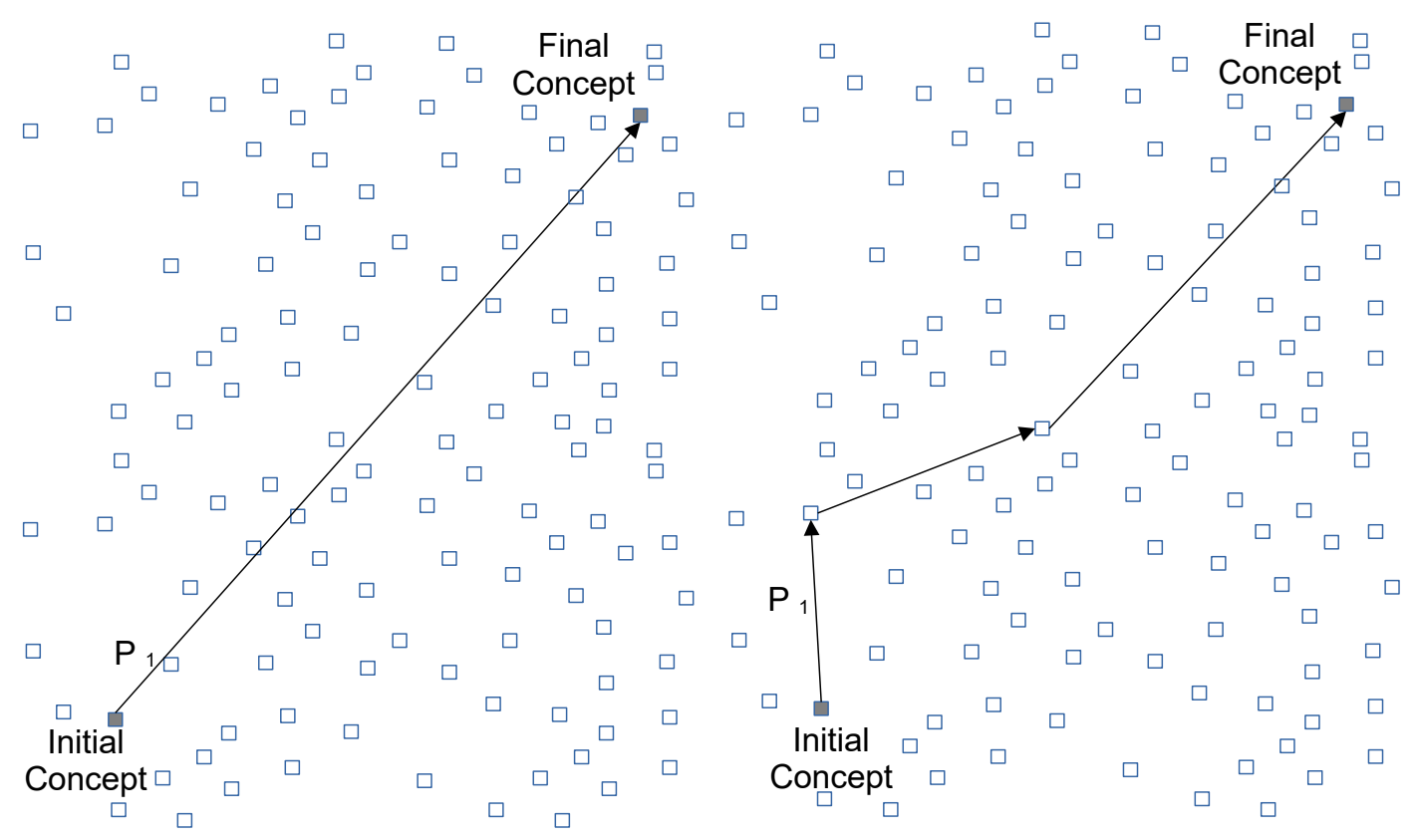

Figure 1. Left: reasoning process using type 1 reasoning. Right: Reasoning process using type 2 reasoning.

Assuming that any computer program or algorithm is an automation of human reasoning, then any pattern recognition based algorithm (machine learning) or any logic based (procedural) computer program can also be seen as the automation of a set of paths through this conceptual space, and can therefore be represented by a set of such paths. Assume it is possible to decouple any such machine learning algorithm or procedural program into a library of functional components that implement those paths or path segments. Assuming that there are multiple functional components implementing a single path, a GCI defines the functionality that is hypothesized to be required to compare the fitness of each functional component in achieving that targeted objective, so that selection of the optimal component can potentially be made from an exponentially larger number of components at exponentially greater speed. Therefore, any procedural program or algorithm can potentially be decoupled into a library of implementations of such paths, where a GCI might be used to significantly increase the group's capacity to leverage the best component of any functionality available anywhere, in order to significantly increase outcomes of computing.

One of the main benefits of Human-Centric Functional Modeling is that all functional state spaces have properties in common, so that understanding one helps to understand all others. Furthermore, the conceptual space is the only functional state space that is inherently ensured to be conceptualizable, 
because it is explicitly based on concepts (the functional states of the cognitive system), instead of other functional states that must be converted to concepts.

Leveraging HCFM to define a functional model of the cognitive system and of the functional state space that describes its behavior, that understanding might then be reused in understanding all other human systems and their behavior, including vision. All other human systems are also represented in HCFM as navigating their own functional state space. Just as procedural programs and text based machine learning algorithms might be decomposed into a library of functional components that each represent a path or paths through the conceptual space, procedural image processing programs and machine learning algorithms for visual pattern recognition might also be decomposed into functional components in this way.

In functional state space a problem is defined as the lack of a path between one functional state and another, that is, the lack of a process for transforming from one functional state to another. A solution is the path through functional state space representing that process. Every model of any process is then a represented by a path through some functional state space, as is every implementation of every process.

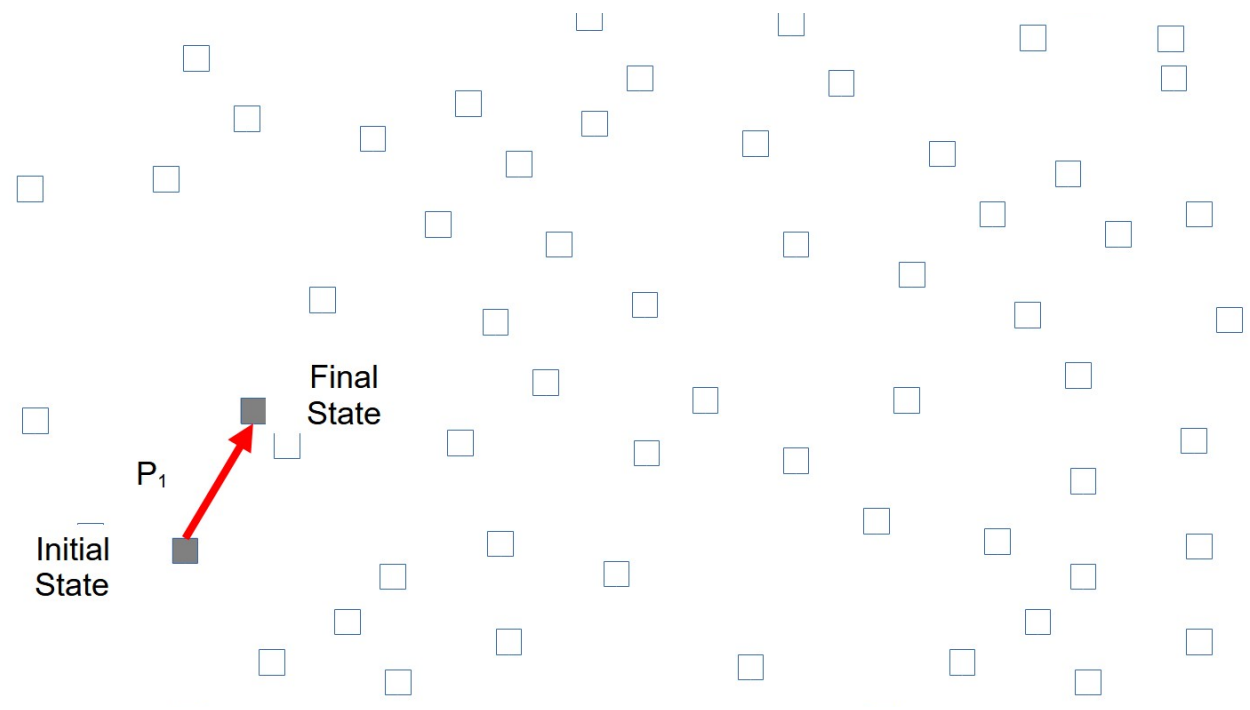

Figure 2. Every process executed by any model of a system can then potentially be represented as a path through a functional state space.

\section{Indexing Libraries of Functional Components in Functional State Space}

In conceptual space a set of concepts is still a concept, and each concept is represented by a geometry. This geometry might enclose a large area representing a generalization of a concept that contains many smaller concepts, or it might enclose a small area representing the specification of a few concepts or of a single larger concept. Reasoning is represented as a path from one geometry representing one concept to another geometry representing another as in figure 3. 


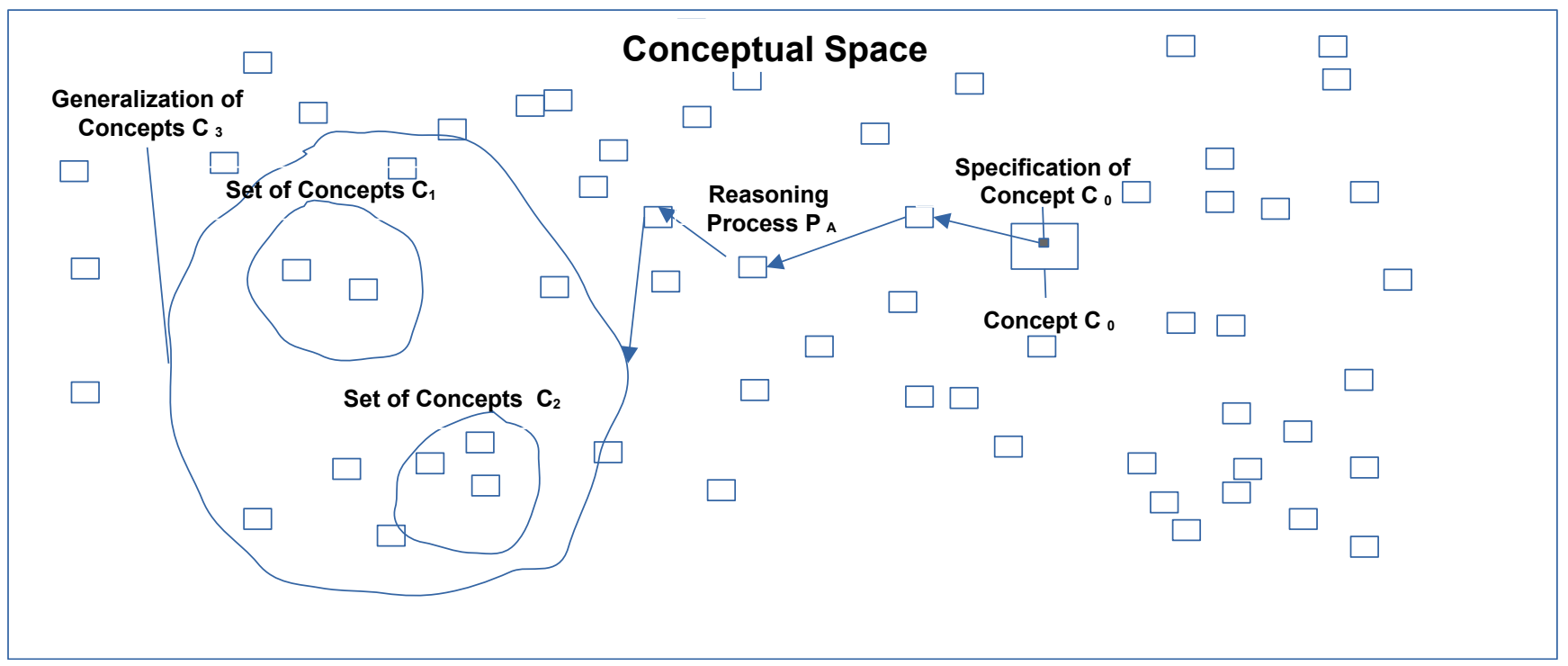

Figure 3. Reasoning is a path from one concept, in this case right to left from a smaller concept within the concept $\mathrm{CO}$ that represents the specification of $\mathrm{CO}$, to a larger concept $\mathrm{C} 3$ that represents the generalization of concepts $C 1$ and $C 2$..

By decoupling each machine learning algorithm or procedural program into a set of processes and their corresponding functional components, those functional components can be added to a library that can be used to increase general problem-solving ability in each functional domain.

\section{Library of Processes}
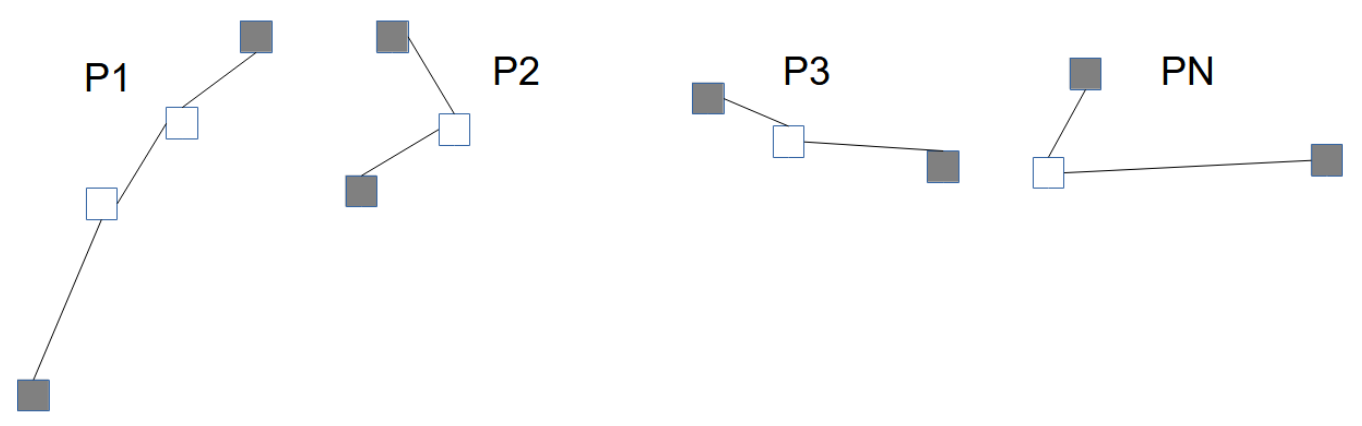

Figure 3. A library of theoretical models of processes as well as of implementations of processes.

We can then define a single measure of fitness by which all such processes might be compared. 


\section{Fitness of Processes}

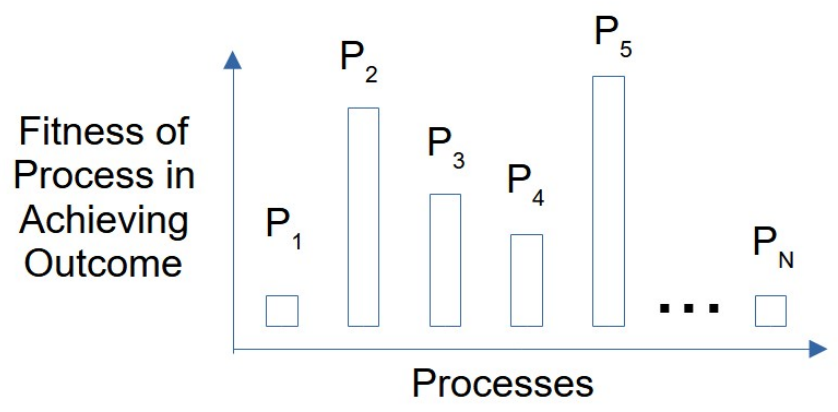

Figure 4. The fitness of each model of each process in a given context of execution can be compared.

This can enable each model to be reused in an exponentially greater number of instances where it is most fit in achieving an outcome, thereby exponentially increasing the group's collective ability to solve problems of understanding systems in each domain.

\section{Modeling Functional Components in Functional State Space}

In conceptual space reasoning occurs within the context of concepts in the region surrounding both the initial concept from which reasoning started and the target concept at which reasoning ends. In the Human-Centric Functional Modeling approach processes in functional state space are represented in terms of the set of functional states they receive as input, the set of functional states they produce as output, and the set of functional states that define the context of execution. As an example, for the function $\mathrm{F}=\mathrm{Ax}+\mathrm{By}$ the values of the variables $[\mathrm{x}, \mathrm{y}]$ are input, and the values of the constants [A, B] form the context of execution.

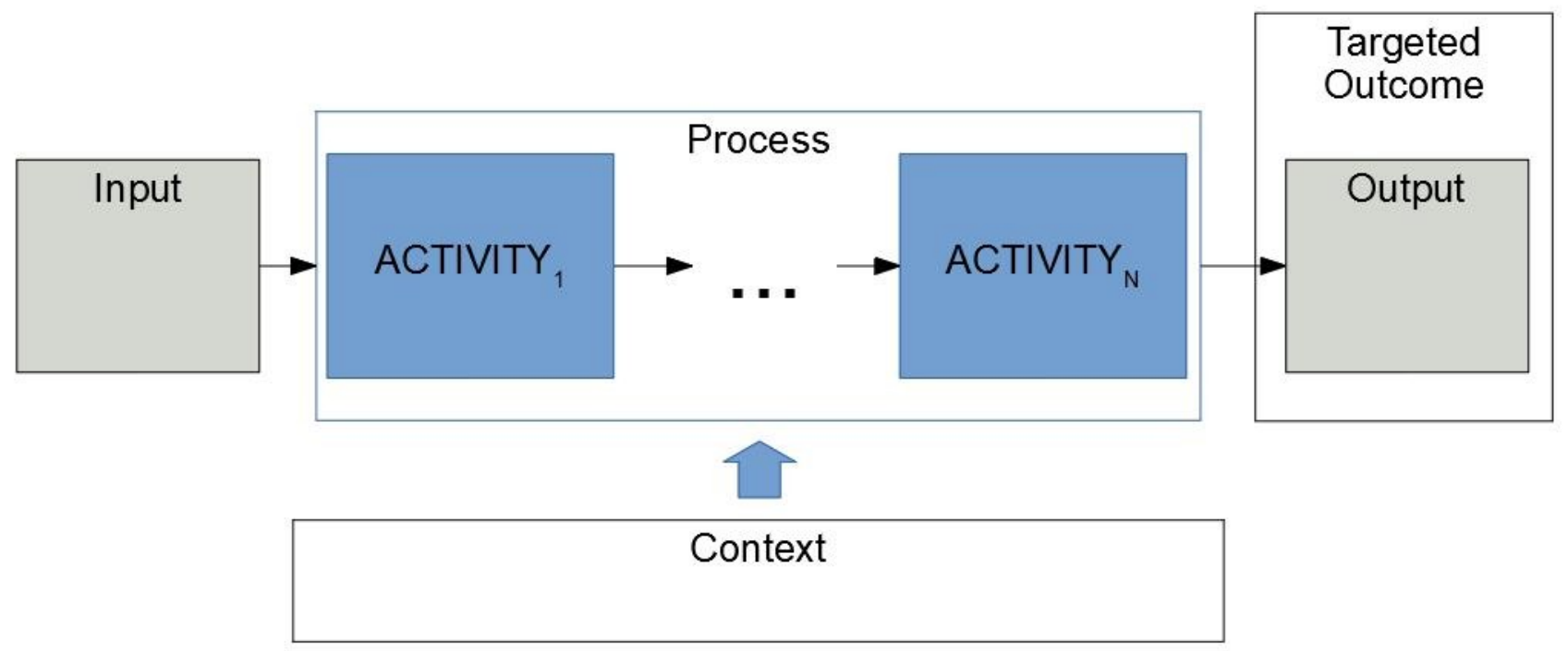

Figure 4. Functional model of a process in a functional state space such as the conceptual space. 
If it is possible to define a "visual state space" in which the geometry and properties of each visual object can be specified within that object itself, then processes in visual state space represent any transformations from one visual object to another. Such transformations might entail transforming (resolving) an image or series of images into recognition of an object. Or such transformations might entail scaling, shifting, or other geometric transforms. In the conceptual space it is hypothesized that any reasoning can be represented as a composition of four basic reasoning functions. These reasoning functions then "span" conceptual space [4]. In the same way, if it is true that all image manipulation operations are representable in the visual state space, it is hypothesized that the visual state space must have a basic set of operations that "spans" the space in that all processes in the visual state space can be represented as a composition of those operations.

Operations in the visual state space however are only intended to represent the manipulation of recognizable objects. It is operations in the visual field that are intended to represent the manipulation of raw image data. Therefore, when mapping visual pattern recognition and other vision related algorithms to the human system using HCFM, it is important to first determine which domain these algorithms as in table 1.

Processes
Object region proposal algorithms [5].
Different imaging processes such as event based
imaging [6].
Human pose estimation algorithms [7].

Human visual feature learning algorithms [8].

Image segmentation [9].

Image resolution improvement [10].

Human action recognition [11].

Dense object detection [12].

\section{Mapping to the Visual Field or Visual State} Space

Hypothesized to convert elements of the visual field to the visual state space.

Hypothesized to involve processing in the visual field.

Hypothesized to involve processing in the visual state space.

Hypothesized to involve processing in the visual state space.

Hypothesized to involve processing in the visual field.

Hypothesized to involve processing in the visual field.

Hypothesized to involve processing in the visual state space.

Hypothesized to involve processing in the visual field.

Table 1. Some processes that must be represented in visual state space.

\section{Limitations}

A number of platforms approximating a GCI, and ontologies within each of those platform that approximate a small region of the collective conceptual space, have already been designed, however neither a complete GCI nor a complete representation of the collective conceptual space have yet been implemented. For this reason, some fundamental properties of functional state spaces remain to be elaborated, such as distances and how they are quantified. Solving these problems in representing one functional state space is expected to solve these problems in representing all other functional state spaces, however they must be solved before a complete implementation of a visual state space can be defined so paths through this space can essentially be used as a unique index in a database of visual processing algorithms. Furthermore, there is the problem of ensuring that all visual processing operations can be represented within this hypothetical visual state space. Though a set of operations to 
span the conceptual space has been proposed, no effort has yet been made to define a set of operations to span the visual state space.

\section{Conclusions}

The collective space of concepts or "collective conceptual space" that represents the functional state space of a GCI is hypothesized to enable understanding rather than just information to be exchanged, so that collective reasoning might occur at vastly greater speed and scale. Similarly, when HCFM is applied to define this hypothetical visual state space the use of GCI is hypothesized to enable recognition of images rather than just visual data to be exchanged, so that collective vision might occur at vastly greater speed and scale as well. Much work remains to validate these ideas, but if valid, this might constitute a significant advance in the field.

\section{References}

[1] Andy E. Williams, Defining a Continuum from Individual, to Swarm, to Collective Intelligence, to General Collective Intelligence, In print (2021), Int. J. Of Collaborative Intelligence, in press (2022)

[2] Andy E. Williams, A Revolution in Systems Thinking?, Proceedings 2021 Congress of the World Organization of Systems and Cybernetics (WOSC), in press (2022)

[3] Kahneman, Daniel. Thinking, fast and slow. Macmillan, 2011.

[4] Williams A.E. (2020) A Model for Artificial General Intelligence. In: Goertzel B., Panov A., Potapov A., Yampolskiy R. (eds) Artificial General Intelligence. AGI 2020. Lecture Notes in Computer Science, vol 12177. Springer, Cham. https://doi.org/10.1007/978-3-030-52152-3 38

[5] S. Ren, K. He, R. Girshick and J. Sun, "Faster R-CNN: Towards Real-Time Object Detection with Region Proposal Networks," in IEEE Transactions on Pattern Analysis and Machine Intelligence, vol. 39, no. 6, pp. 1137-1149, 1 June 2017, doi: 10.1109/TPAMI.2016.2577031.

[6] G. Gallego et al., "Event-Based Vision: A Survey," in IEEE Transactions on Pattern Analysis and Machine Intelligence, vol. 44, no. 1, pp. 154-180, 1 Jan. 2022, doi: 10.1109/TPAMI.2020.3008413.

[7] Z. Cao, G. Hidalgo, T. Simon, S. -E. Wei and Y. Sheikh, "OpenPose: Realtime Multi-Person 2D Pose Estimation Using Part Affinity Fields," in IEEE Transactions on Pattern Analysis and Machine Intelligence, vol. 43, no. 1, pp. 172-186, 1 Jan. 2021, doi: 10.1109/TPAMI.2019.2929257.

[8] L. Jing and Y. Tian, "Self-Supervised Visual Feature Learning With Deep Neural Networks: A Survey," in IEEE Transactions on Pattern Analysis and Machine Intelligence, vol. 43, no. 11, pp. 40374058, 1 Nov. 2021, doi: 10.1109/TPAMI.2020.2992393.

[9] L. -C. Chen, G. Papandreou, I. Kokkinos, K. Murphy and A. L. Yuille, "DeepLab: Semantic Image Segmentation with Deep Convolutional Nets, Atrous Convolution, and Fully Connected CRFs," in IEEE Transactions on Pattern Analysis and Machine Intelligence, vol. 40, no. 4, pp. 834-848, 1 April 2018, doi: 10.1109/TPAMI.2017.2699184.

[10] C. Dong, C. C. Loy, K. He and X. Tang, "Image Super-Resolution Using Deep Convolutional Networks," in IEEE Transactions on Pattern Analysis and Machine Intelligence, vol. 38, no. 2, pp. 295307, 1 Feb. 2016, doi: 10.1109/TPAMI.2015.2439281. 
[11] S. Ji, W. Xu, M. Yang and K. Yu, "3D Convolutional Neural Networks for Human Action Recognition," in IEEE Transactions on Pattern Analysis and Machine Intelligence, vol. 35, no. 1, pp. 221-231, Jan. 2013, doi: 10.1109/TPAMI.2012.59.

[12] T. -Y. Lin, P. Goyal, R. Girshick, K. He and P. Dollár, "Focal Loss for Dense Object Detection," in IEEE Transactions on Pattern Analysis and Machine Intelligence, vol. 42, no. 2, pp. 318-327, 1 Feb. 2020, doi: 10.1109/TPAMI.2018.2858826. 